See Article page 1719.

\section{Commentary: Trauma alert! Evidence to support the safe use of contused lungs}

\author{
Lara Schaheen, MD, ${ }^{\mathrm{a}}$ and \\ Jonathan D'Cunha, $\mathrm{MD}, \mathrm{PhD}^{\mathrm{b}}$
}

Lung transplantation (LTx) is the best option for patients with end-stage lung disease when medical therapy has run its course. Achieving outstanding 3- and 5-year results is a challenging multifactorial process that includes appropriate donor selection. Although there are a limited number of suitable donor lungs, polytrauma donors have traditionally been used very selectively due to the unknown downstream issues with injured lungs that exhibit contusions. Hesitation in using these donors is in large part due to the unknown consequences: primary graft dysfunction (PGD), ventilator-associated pneumonia, early graft rejection, and chronic lung allograft dysfunction.

In this issue of the Journal, Schwarz and colleagues ${ }^{1}$ and the LTx group in Vienna retrospectively reviewed 1152 donors that had a history of polytrauma over an 8-year period. Donors reviewed included those with injuries to multiple areas of the body including injuries to the chest. The authors' study sought to evaluate the safety of use of these donors. The authors should be commended for tackling this issue, and this report brings forth interesting data that would benefit from further refinement moving forward.

In their study, they analyzed donors by dividing them into 3 groups: polytrauma with contusions (radiographic evidence), polytrauma clear (no radiographic signs of contusion) at the time of organ offer, and a control group (nontrauma donor lungs). The vast majority of contusions were diagnosed by computed tomography (75\%), and $54 \%$ of the donors had bilateral contusions. In total, $54 \%$ of donors had injury restricted to 1 or 2 lobes. Surprisingly,

\footnotetext{
From ${ }^{a}$ Norton Thoracic Institute, St Joseph's Hospital and Medical Center; and ${ }^{\mathrm{b}}$ Department of Cardiothoracic Surgery, Mayo Clinic Arizona, Phoenix, Ariz.

Disclosures: The authors reported no conflicts of interest.

The Journal policy requires editors and reviewers to disclose conflicts of interest and to decline handling or reviewing manuscripts for which they may have a conflict of interest. The editors and reviewers of this article have no conflicts of interest.

Received for publication Nov 23, 2020; revisions received Nov 23, 2020; accepted for publication Nov 23, 2020; available ahead of print Dec 3, 2020.

Address for reprints: Jonathan D'Cunha, MD, PhD, Department of Cardiothoracic Surgery, Mayo Clinic Arizona, 5777 E Mayo Blvd, Phoenix, AZ 85054 (E-mail: DCunha.Jonathan@mayo.edu).

J Thorac Cardiovasc Surg 2022;163:1737-8

$0022-5223 / \$ 36.00$

Copyright (c) 2020 by The American Association for Thoracic Surgery

https://doi.org/10.1016/j.jtcvs.2020.11.120
}
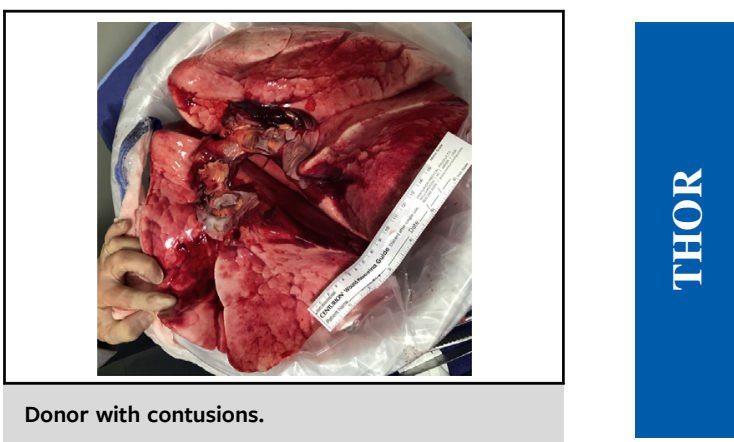

CENTRAL MESSAGE

A large study highlights the potential safety of using lungs from donors with chest trauma. The presence of pulmonary contusions in a lung donor appears not to be associated with worse outcomes.

at least $25 \%$ of the patients in the contusion group only had a chest radiograph for their donor evaluation. The authors found that there was no difference in grade 3 PGD at 72 hours, length of ventilation, short-term survival, and long-term graft survival. Time to best forced expiratory volume in 1 second was not impacted significantly. Propensity matching was used to address differences in the baseline donor characteristics, as the polytrauma donors tended to be younger and male predominant. Results from the analysis of the matched groups confirmed that no difference was found between groups. In addition, freedom from chronic lung allograft dysfunction $\geq 1$ was similar between the matched groups.

The study is a clear step forward. We believe the strength in this study lies in the institutional experience and the numbers. The major limitations of the study are rooted in selection bias. The donor lungs evaluated in this series had already passed standard evaluation parameters. Thus, the retrospective review of donor lungs that have already been deemed adequate for transplantation is of unclear significance. Pulmonary contusions vary in severity, anatomic distribution, and progression. What was the quality assurance of true contusion? Furthermore, there was no real or standardized scoring of severity. What was the influence of transfusion as a more finite and quantitative variable in these donors? Were there predictors of donor quality that should be looked at in more detail, such as airway pressures and other measures of compliance? 
A review of the operative approach for lung transplantation used in this study should be highlighted. This is especially important as others apply these findings to their own programs. The intraoperative use of venoarterial extracorporeal membrane oxygenation is something that we have performed since 2013 and is the Vienna groups' routine approach. ${ }^{2}$ This affords controlled reperfusion/perfusion throughout the entire procedure as well as protective ventilation during this critical time. The authors have the lowest reported rates of PGD in the literature using this approach and this should not be overlooked as part of this analysis.

This study suggests that donor lungs with contusions can be used if function is good without jeopardizing short- and long-term outcomes. Moving forward, a more analytical approach should be taken to quantify the extent of contusion to further expand upon this important work. Contusion is not a binary variable, but we would put forth this variable to be considered along a continuum in relationship to evaluating donor lungs for LTx. Contusion is not only a radiographic finding but should also be coupled with ventilatory and functional parameters for true assessment. Bringing these studies forward will be critical to understanding ways in which we can increase the supply of suitable donor lungs for the future. There is no more important time for these steps forward as the denominator of patients with lung failure who will need transplantation as a result of disease progression from our current coronavirus disease 2019 (COVID-19) pandemic and other pathologic mechanisms of lung failure will most assuredly increase.

\section{References}

1. Schwarz S, Rahimi N, Kifjak D, Frommlet F, Benazzo A, Jacksch P, et al. Lungs from polytrauma donors with significant chest trauma can be safely used for transplantation. J Thorac Cardiovasc Surg. 2022;163:1719-31.e2.

2. Hoetzenecker K, Benazzo A, Stork T, Sinn K, Schwarz S, Schweiger T, et al. Bilateral lung transplantation on intraoperative extracorporeal membrane oxygenator: an observational study. J Thorac Cardiovasc Surg. 2020;160:320-7.e1. 